\title{
Immobilized Carbon Nanotubes as Matrix for MALDI-TOF-MS Analysis: Applications to Neutral Small Carbohydrates
}

\author{
Shi-fang Ren, Li Zhang, Zhi-hong Cheng, and Yin-long Guo \\ Shanghai Mass Spectrometry Center, Shanghai Institute of Organic Chemistry, Chinese Academy of Sciences, \\ Shanghai, People's Republic of China
}

In this work, we reported on the advantages of immobilized carbon nanotubes as a novel MALDI-matrix. Recently, carbon nanotubes have been reported to be an effective MALDI matrix for small molecules (Anal. Chem. 2003, 75, 6191), as it can eliminate the interfering matrix peaks as well as form a web morphology to fully disperse the analyte and allow strong ultraviolet absorption for enhanced pulsed laser desorption and ionization. In our study, to overcome the problem that the carbon nanotube matrix may fly off from the target, a type of polyurethane adhesive, NIPPOLAN-DC-205, is introduced to immobilize carbon nanotubes on the target, which enables widespread application of carbon nanotubes as matrix for MALDI-MS analysis. At the same time, the properties of the carbon nanotubes as an efficient matrix remained after immobilization. The presence of NIPPOLAN-DC-205 increases the time for analysis at a particular desorption spot by minimizing the time-consuming search for "hot spots" and facilitating experiments such as post source decay (PSD) which need longer-lasting signals. Moreover, NIPPOLAN-DC-205 produces no interference peaks and can easily be cleaned with acetone. Fast evaporation technology may be used to enhance signal reproducibility in MALDI analysis using carbon nanotubes as matrix. Consequently, the applicability of the carbon nanotube as matrix for matrixassisted laser desorption/ionization mass spectrometry (MALDI-MS) analysis of low molecular mass analytes is highly improved. The feasibility of the method employing polyurethane is demonstrated by comparison of the results produced from the carbon nanotube matrix with and without immobilization. In addition, neutral small carbohydrates, which are difficult to be ionized normally, can be cationized with high efficiency by MALDI-TOF-MS using the immobilized carbon nanotube matrix. The method was further applied to analyze peptides and detect urine glucose successfully. (J Am Soc Mass Spectrom 2005, 16, 333-339) (c) 2005 American Society for Mass Spectrometry

$\mathrm{M}$ atrix-assisted laser desorption/ionization mass spectrometry (MALDI-MS) developed by Karas and Hillenkamp [1-3] has become a versatile and important tool for the determination of molecular masses of various fragile and nonvolatile samples such as proteins and carbohydrates $[2,4,5]$. Some success in the use of MALDI-TOF MS for the characterization of many low molecular weight compounds has been reported [6-11]. However, there is a valid concern that analysis of small molecules, especially those with molecular weights in the matrix region $(m / z<500)$, can be subjected to interference with matrix peaks.

For this reason, different matrix substances, such as porphyrins [12, 13], inorganic material [14-17], fullerene [18, 19], and porous silicon [20], have also

Published online January 14, 2005

Address reprint requests to Dr. Y-L. Guo, Shanghai Mass Spectrometry Center, Shanghai Institute of Organic Chemistry, Chinese Academy of Sciences, Shanghai 200032, People's Republic of China. E-mail: ylguo@ mail.sioc.ac.cn been used to eliminate matrix ion interference. Recently, carbon nanotubes as an alternative to conventional organic matrices were utilized successfully for low molecular mass analytes of peptides, organic compounds, and $\beta$-cyclodextrin [21]. Carbon nanotubes can transfer energy to the analyte under laser irradiation, which causes analytes to be well desorbed and ionized. Further, the interference of matrix ions can be eliminated and the fragmentation of the analyte avoided while maintaining good sensitivity and excellent reproducibility [21]. These qualities of the carbon nanotube mentioned above makes it an attractive candidate for matrix for analysis of low molecular weight compounds.

However, employing carbon nanotubes as matrix has the limitation that carbon nanotubes may fly off from the target when subjected to a pulse from a laser. This may result in some disadvantages in the use of nanotubes; for example, contaminating the mass spectrometer, obtaining time-limited signals, and time-consuming searching for "hot spots". However, perform- 
ing sample deposition method such as fast evaporation technology [22] or electrospray deposition (ESD) [23], which may improve sample homogeneity, is difficult because the carbon nanotube matrix will be blown off during deposition process. Thus, the applicability of carbon nanotubes as a matrix for MALDI may be limited. To overcome this problem, we are seeking an approach to immobilize carbon nanotubes on the target. Adhesive tape has often been used to immobilize inorganic material such as graphite particles and porous silicon [18, 20]. Sunner and Han [18] introduced activated carbon as a matrix for desorption/ionization of proteins and peptides. In their experiment, they attempted to solve the problem of contamination of the ion source by suspended particles. The addition of glycerol was expected to make the carbon particles to be more "sticky" and ensured that the ion signals could be obtained for extended periods of time. However, glycerol can also introduce interference peaks by itself. In our study, a sort of polyurenthane adhesive, NIPPOLLAN-DC 205, which was prepared from isocyanates and polyester polyols, was introduced for the direct tight bonding of the carbon nanotube on the steel target through interfacial strength. The introduction of NIPPOLAN-DC-205 does not produce interference peaks and it is easy to wipe off the immobilized nanotubes with acetone.

It has long been recognized by the MALDI community that the direct analysis of neutral carbohydrates is difficult owing to poor carbohydrate response. This is because carbohydrates lack basic sites to which a proton could attach, with the result that carbohydrates become ionized instead by the addition of metal ions, usually $\mathrm{Na}^{+}$, with low efficiency [5, 24]. Mass spectrometry, specifically with fast atom bombardment (FAB) and liquid secondary ion mass spectrometry (LSIMS), was also extensively used to analyze carbohydrates [25-29], but they gave relatively poor detection limits. Therefore, derivatization is often an essential stage for carbohydrates analysis. MALDI-MS has also been used to analyze carbohydrates. However, for the analysis of small carbohydrates, the matrix (such as DHB) interferes heavily and sensitivity is relatively low [30,31]. Gouw et al. [30] reported that the sensitivity can be increased by a factor of 1000 by the addition of a quaternary ammonium center.

In this work, we demonstrate that the aforementioned limitations of nonimmobilized carbon nanotubes applied as MALDI-matrix were overcome successfully. The applicability of carbon nanotubes as MALDI-matrix by immobilization with polyurethane adhesive was supported by a series of experiments. It was also found that direct analysis of neutral small carbohydrates, which are difficult to be ionized normally, was conveniently performed using immobilized carbon nanotubes as matrix. We also reported extending the method to analyze peptides and detect glucose in urine samples.

\section{Experimental}

\section{Chemicals and Materials}

All neutral carbohydrates, including xylose, arabinose, ribose, mannose, glucose, fructose, galactose, sucrose, cellobiose, maltose, lactose, maltopentaose and $\alpha$-cyclodextrin $(\alpha-\mathrm{CD})$, which were HPLC grade, were obtained from Fluka Chemie $\mathrm{GmbH}$, Buchs, Switzerland. The multi-walled carbon nanotube particles used in the study were obtained from Sun Nanotech (Shanghai, China). HPLC grade methanol and MilliQ water (Millipore, ELPaso, TX) were used in our experiments. NIPPOLLAN-DC 205 was purchased from Nippon Polyurethane Industry (Tokyo, Japan). Polyurethane adhesives, including adhesive P1, P2, P3 and P4 were obtained from Shanghai Jiuyuan Petrochemical Co. Ltd. (Shanghai, China). Fresh urine samples were collected from a diabetic patient and a healthy man. The other chemical reagents were of analytical grade and were used without further purification.

\section{Sample Preparation}

The carbon nanotubes were washed successively with acetone, methanol, $1 \% \mathrm{NaOH}$ aqueous solution, $0.4 \% \mathrm{HCl}$ aqueous solution, and water. Then, $2 \mathrm{mg}$ of carbon nanotube was suspended in $1 \mathrm{ml}$ of water/methanol (1:1, $\mathrm{vol} / \mathrm{vol}$ ) by sonication for a few minutes.

All the monosaccharides, disaccharides, and $\alpha-\mathrm{CD}$ were dissolved in water/methanol (3:2, vol/vol) at a concentration of $1 \times 10^{-3} \mathrm{M}$. Polyurethane adhesives were diluted with ethyl acetate $(0.2 \mathrm{~g} / 1 \mathrm{ml})$.

The urine sample was deproteinized with trichloroacetic acid and centrifuged at 10,000 rpm for $10 \mathrm{~min}$ before analysis.

\section{MALDI Analysis}

About $1 \mu$ l polyurethane adhesives solution was applied to a stainless steel target probe. Then $1.5 \mu \mathrm{l}$ carbon nanotube suspension solution was pipetted on top of the NIPPOLLAN-DC 205 layer and allowed to dry at room temperature. The sample solution, such as monosaccharides, disaccharides, $\alpha-\mathrm{CD}$ and urine was deposited on the carbon nanotube layer and dried at ambient conditions. The fast evaporation method was performed as reported previously [22].

\section{Instrument Setup}

MALDI-TOF-MS analyses were performed employing delayed extraction in positive ion mode on a time-offlight mass spectrometer (Voyager- DE STR, Applied Biosystems, Framingham, MA) with a $2.0 \mathrm{~m}$ flight tube. Desorption/ionization was obtained by using a $337 \mathrm{~nm}$ nitrogen laser with a 3 ns pulse width. Available accelerating potential is in the range of $+20 /-20 \mathrm{kV}$. The actually applied acceleration voltage in our exper- 

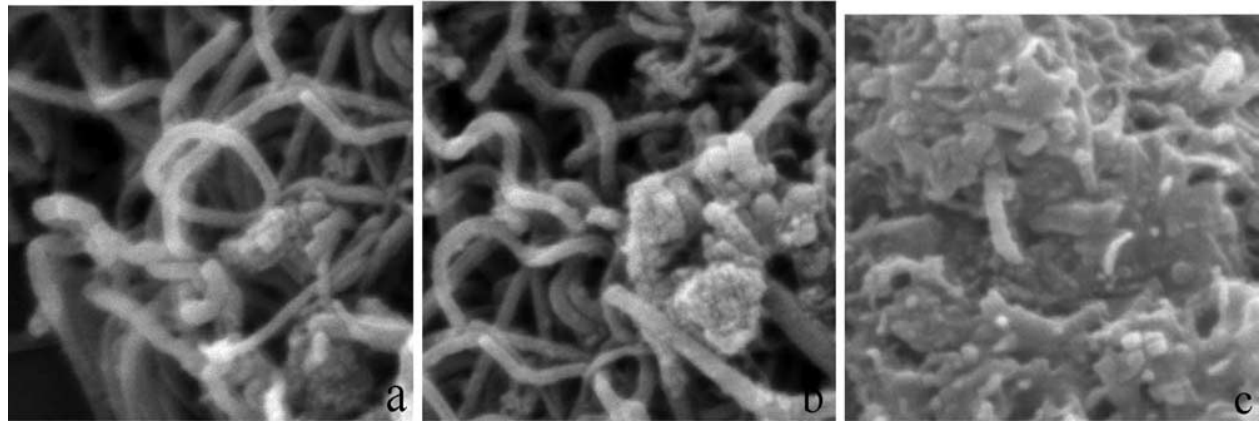

Figure 1. SEM images $(\times 20,000$-fold $)$ of (a) multi-walled carbon nanotube layer in the absence of NIPPOLAN-DC 205 before dropping sample solution, (b) multi-walled carbon nanotube layer in the presence of NIPPOLAN-DC 205 before dropping sample solution, (c) multi-walled carbon nanotube layer in the presence of NIPPOLAN-DC 205 after dropping sample solution.

iment was $20 \mathrm{kV}$. Laser power was adjusted to slightly above the threshold to obtain good resolution and signal-to-noise ratios, and mass calibration was achieved using DHB as an external standard (about $0.05 \%$ mass accuracy can be achieved). In the PSD experiment, the precursor ions (sodium adducts) were isolated using a timed ion selector. The spectra shown generally represent the sums of 50 laser shots, unless otherwise stated. Examination and processing of mass spectral data was performed by Data Explorer 4.0 software supplied by Applied Biosystems.

\section{Results and Discussion}

\section{The feasibility of the Method}

Of the polyurethanes used, only NIPPOLAN-DC 205 gave satisfactory results. The other adhesives (P1, P2, $\mathrm{P} 3$, and $\mathrm{P} 4$ ) produced interference peaks and very weak or almost no analyte signals at all could be observed. The different results from the five adhesives may be attributable to different chemical and physical properties. Compared to the other four adhesives, NIPPOLAN-DC 205 has a higher molecular weight, higher vapor pressure, and a more stable structure. Thus NIPPOLAN-DC 205, which was prepared from isocyanates and polyester polyols (MW >30,000), was selected to immobilize the carbon nanotube on the metal target through interfacial interaction for the following reasons: it is stable under our experiment conditions, it offers very clean background, and it can easily be cleaned from the target with acetone.

Figure 1 shows SEM images of (Figure 1a) carbon nanotube layer in the absence of NIPPOLAN-DC 205 before dropping sample solution, (Figure 1b) carbon nanotube layer in the presence of NIPPOLAN-DC 205 before dropping sample solution, (Figure 1c) carbon nanotube layer in the presence of NIPPOLAN-DC 205 after dropping sample solution. Comparing SEM image results shown in Figure 1a and b, we can see that there is almost no change in the fibrelike texture of carbon nanotubes with and without NIPPOLAN-DC 205. As shown in Figure 1a and b, the carbon nanotube layer before depositing the sample solution appears to have web morphology to disperse analytes. However, as is clear from the SEM image of the carbon nanotube layer after deposition of the sample solution, the web morphology was masked as a result of the sample molecules being absorbed onto the surface of the carbon nanotube layer. It can be seen that the immobilized carbon nanotube matrix provides a medium for crystallization of the sample molecules, which is in agreement with previous reports of the not-immobilized carbon nanotube matrix [21].

To test whether immobilized carbon nanotubes are suitable as matrix for MALDI-MS, we collected a mass spectrum on a blank spot on which polyurethane adhesive was dropped, followed by the carbon nanotube suspension. Almost no signals from NIPPOLAN-DC 205 and matrix were visible by positive ion MALDI-MS. Figure $2 \mathrm{a}$ and $\mathrm{b}$ show the mass spectra of equal amounts of glucose using the immobilized and notimmobilized carbon nanotube as matrix respectively. Intense ion signals due to sodium ion adduct $(\mathrm{M}+$ $\mathrm{Na})^{+}$and potassium ion adduct $(\mathrm{M}+\mathrm{K})^{+}$at $m / z 203$ and 219 were observed on both mass spectra. No interference peak from the polyurethane adhesive was observed. It appeared from the observed signal strengths that the efficiency of the matrix for desorption/ionization of glucose was almost the same with or without the adhesive. Thus, properties such as its strong ultraviolet absorption, which is essential for a successful matrix, did not change after immobilization by NIPPOLAN-DC 205.

The other eleven carbohydrates, including xylose, ribose, arabinose, fructose, mannose, galactose, sucrose, cellobiose, maltose, lactose, and maltopentos, were analyzed and intense peaks corresponding to each carbohydrate were obtained. The yielded high signal intensities may result from a possibly high ionization efficiency of the immobilized carbon nanotube matrix and from the absence of signal suppression by interfering matrix peaks. Figure $3 a, b, c$, and d show the mass spectra of ribose, fructose, cellobiose, and maltopentaose being representative for pentoses, hexoses, disac- 

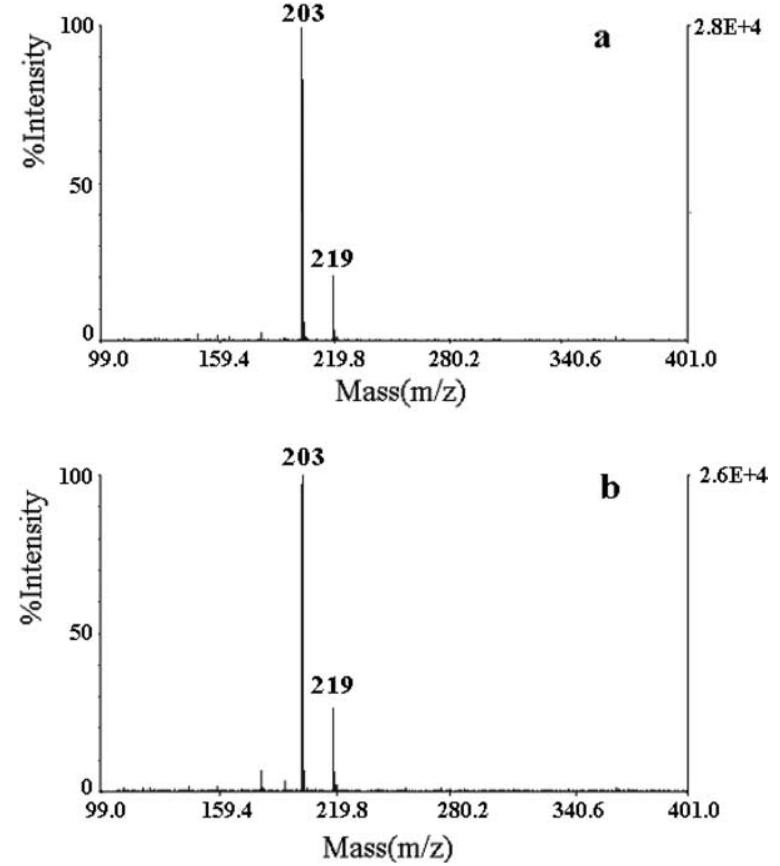

Figure 2. MALDI-TOF mass spectra of glucose using (a) immobilized carbon nanotubes and (b) not-immobilized carbon nanotubes as matrix.

charides and larger noncyclic oligosaccharides, respectively. We found that all carbohydrates produced intense $\mathrm{MNa}^{+}$species as the major ion often accompa-

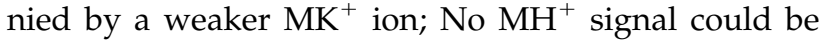
detected, which is due to the low proton affinity of saccharides $[5,32]$.

Another example is the analysis of peptide (Asp-Arg-
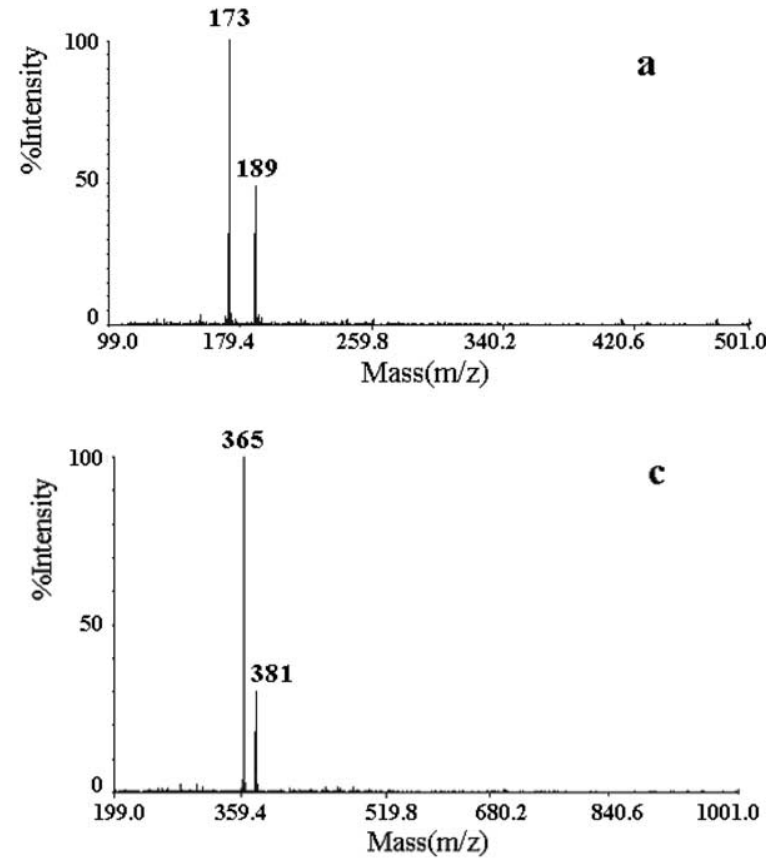

Val-Tyr-Ile-His-Pro-Phe, MW = 1046.2 Da) by MALDITOFMS with immobilized carbon nanotubes as matrix. The obtained mass spectrum is shown in Figure 4. It can be seen that the peptide is ionized as alkali cation adducts. Obviously, the immobilized carbon nanotubes have great potential for application in the detection of a variety of low molecular weight species.

\section{The advantage of the Method}

The utilization of NIPPOLLAN-DC 205 ensured that ion signals are obtained for an extended period of time. Figure 5 shows a direct comparison of carbon nanotube matrix immobilized and not immobilized. It indicates the signal-to-noise ratios of analyte ion signals from a particular and changeless position with MALDI analysis of equal amounts of glucose. Each mass spectrum was obtained by applying 8 laser shots at a particular position in the probe well. And we collected a continuous 20 mass spectra for each position. Such procedure was applied to another 9 positions, and average of the 10 positions afforded the results. As shown in Figure 5, the immobilized carbon nanotube matrix produces higher than average signal-to-noise ratios and longer lasting signals than the not-immobilized matrix. In our experiment, the glucose signal from the particular location can still be observed after acquiring 50 mass spectra continuously when using the immobilized carbon nanotube matrix, while no signal can be obtained after acquiring 10 mass spectra using the not-immobilized matrix. Hence, the immobilized carbon nanotube matrix is preferred in PSD experiments.
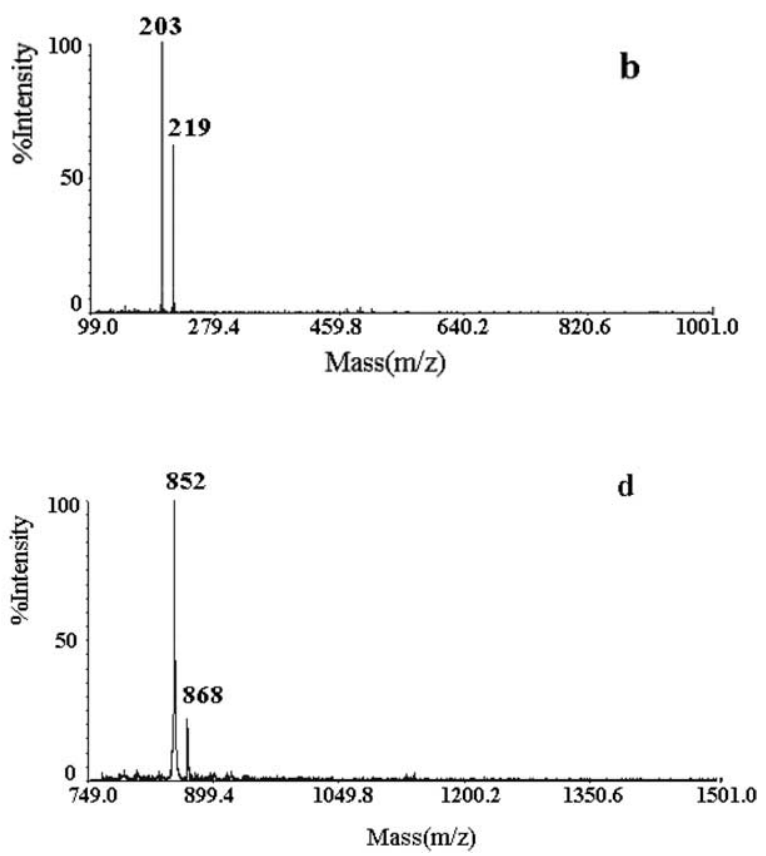

Figure 3. MALDI-TOF mass spectra of (a) ribose, (b) fructose, (c) cellobiose, and (d) maltopentaose, using immobilized carbon nanotubes as matrix. 


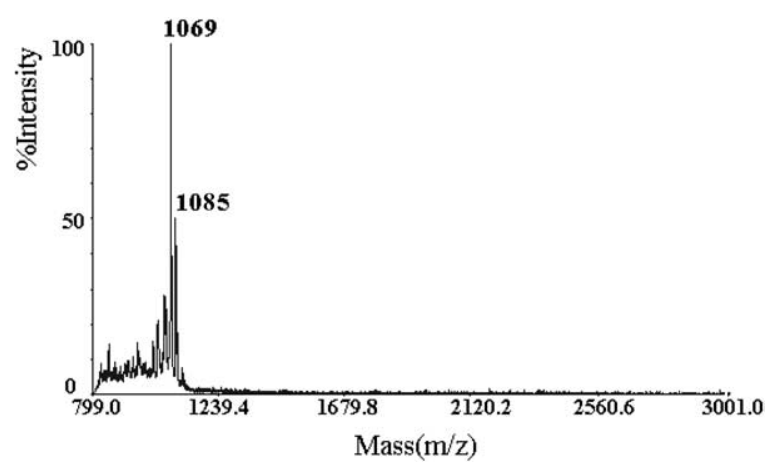

Figure 4. MALDI-TOF mass spectra of peptide (Asp-Arg-ValTyr-Ile-His-Pro-Phe, MW = 1046.2 Da) using immobilized carbon nanotubes as matrix.

Longer-lasting signals favor PSD experiments Figure 6 shows the PSD-MALDI mass spectrum for $[\alpha-\mathrm{CD}+\mathrm{Na}]^{+}$ $(\mathrm{m} / \mathrm{z}$ 995) using the immobilized carbon nanotube as matrix. The PSD experiment can be easily performed using the immobilized carbon nanotube as the matrix, while in the case of the not-immobilized matrix, sample depletion is too rapid. PSD fragment ions $m / z$ 185, 347, 509,671 , and 833 resulted from glycosidic bond cleavage, which may provide structure information of the oligosaccharide.

In addition, fast evaporation technology and ESD can be used when the carbon nanotube was immobilized by NIPPOLAN-DC-205; these techniques are useful to improve quantitative analysis of small molecular weight samples. Here we employ the fast evaporation deposition method using nitrogen gas. The experiment was performed as reported previously [22]. It was found that the immobilized carbon nanotube matrix was not blown off during nitrogen gas spraying. Signal reproducibility was monitored by comparing the signal intensity from fast evaporation deposition and the dried droplet deposited samples. The fast evaporation deposition method produced more uniform analyte ion signal intensities across the spot.

The sensitivity of the MALDI-analysis using immobilized carbon nanotubes as matrix was tested using fructose as analyte prepared in different concentrations.

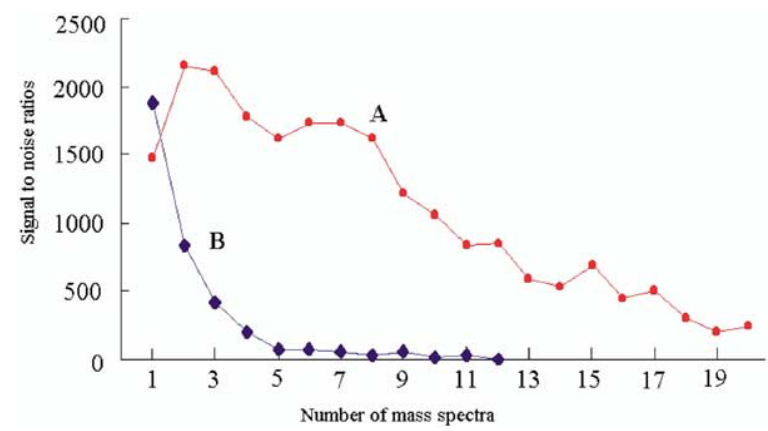

Figure 5. Comparison of signal-to-noise ratio of analyte ion signal in a particular position of probe well with the matrixes of (a) immobilized carbon nanotubes and (b) not-immobilized carbon nanotubes.

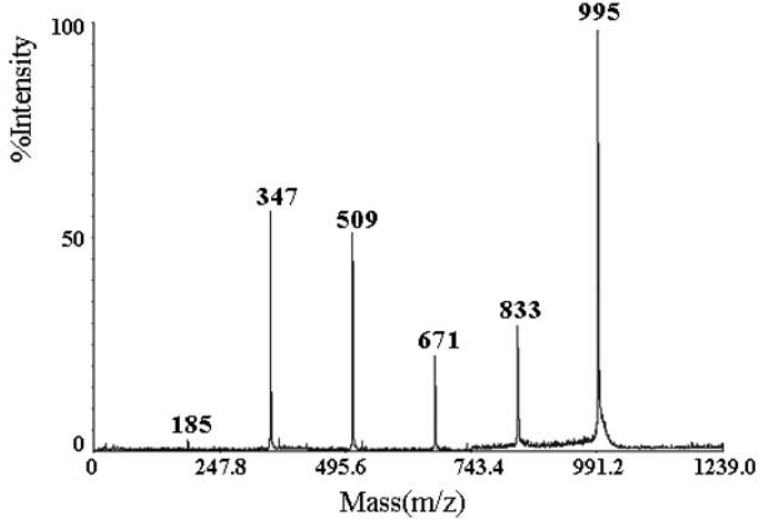

Figure 6. PSD spectrum of $[\alpha-\mathrm{CD}+\mathrm{Na}]^{+}(m / z$ 995). Fragment ions at $\mathrm{m} / \mathrm{z} 185,347,509,671$, and 833 resulted from glycosidic bond cleavage.

The stock solution of fructose was prepared at a concentration of $1 \times 10^{-3} \mathrm{M}$. It was diluted with water/ methanol (vol/vol 3/2) by a 1:50 volumetric ratios and repeated 10 times, and then $0.5 \mu \mathrm{l}$ of the solutions with different concentrations were deposited on the carbon nanotube layer. The results show that about 5 attomol amount analyte could be well detected (signal-to-noise ratio greater than three for the sodium glucose ion adduct) with immobilized carbon nanotubes as matrix, as shown in Figure 7.

The NIPPOLAN-DC 205 forms a thin layer between the metal target and the carbon nanotube layer, which acts like a coating on the target. In such a situation the matrix and analytes have less opportunity to be absorbed into the target. As a consequence this coating may play a role in eliminating potential carry-over effects from the carbon nanotube and analytes. As it takes a rather long time to investigate whether there are carry over effects, this is only a hypothesis and further experiments in the future are needed to support it. In addition, the layer can easily be removed by washing with an acetone solution. Moreover, this method is simple and convenient.

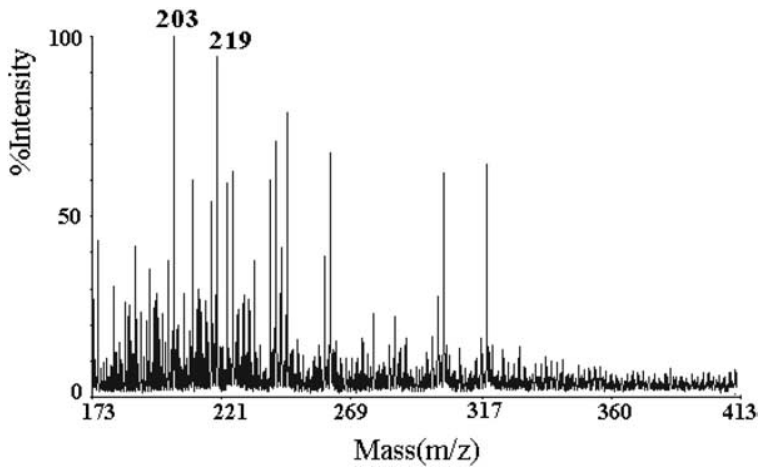

Figure 7. MALDI-TOF mass spectra for sensitivity analysis (5 attomol) with fructose as analyte using immobilized carbon nanotubes as matrix. 

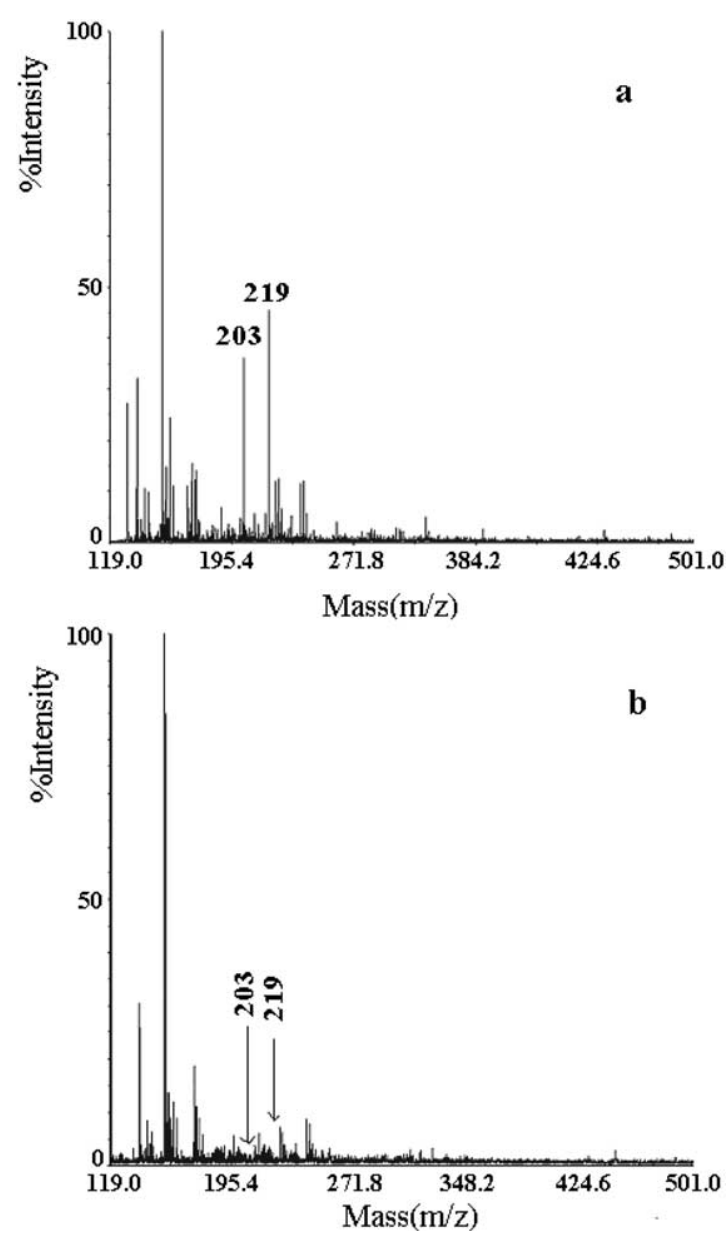

Figure 8. MALDI-TOF mass spectra for urine sample of (a) diabetic patient and (b) healthy man. Unlableled peaks arose from normal urine sample. Ion peaks at $\mathrm{m} / \mathrm{z} 203$ and 219 correspond to urine glucose. Ion peaks for urine glucose were observed on the spectrum for the diabetic patient, while there was no visible glucose signal on the spectrum for the healthy man.

\section{Urine Glucose Analysis}

The ability of immobilized carbon nanotubes for desorption/ionization of analytes was further tested with urine as a mixture sample. Figure 8 shows the mass spectrum of the urine sample from a diabetic patient whose urine glucose level was about $56 \mathrm{mmol} / \mathrm{L}$ tested with reagent strips. Ion peaks at $\mathrm{m} / \mathrm{z} 203$ and 219 corresponding to glucose were observed on the spectrum for the urine of patient, while there was no visible glucose signal on the spectrum for the healthy man. Comparing results from Figure $8 \mathrm{a}$ and $8 \mathrm{~b}$, we can see all unlabeled peaks in Figure 8aöroseđ̛romöheäompounds in normal urine sample. It is therefore hoped that the method presented here will be suitable for routine urine assays in clinical studies and helpful for the diagnosis of urine glucose.

\section{Conclusions}

A simple method is introduced to improve the applicability of carbon nanotube as matrix for analysis of low mass weight compounds by employing a type of polyurethane adhesive, NIPPOLAN-DC 205, which is efficient for bonding carbon nanotubes on the target through interfacial strength. The disadvantage of the original procedure, namely that the carbon nanotube may fly off from the target, was overcome successfully by addition of polyurethane. The immobilized carbon nanotube matrix conserved the properties of carbon nanotubes as an efficient matrix and provided several improvements over the not-immobilized carbon nanotube matrix: these include the avoidance of contamination of the mass spectrometer, increased analyte signal durability together with increased signal to noise ratio, greater reproducibility, absence of interfering matrix peaks in the low mass region, and ease of use of the method. The applicability of immobilized carbon nanotubes as an improved MALDI matrix was successfully demonstrated in the analysis of a set of small carbohydrate molecules like pentoses, hexoses, and dihexoses as well as in the direct analysis of glucose in human urine.

\section{Acknowledgments}

The authors gratefully acknowledge funds provided by the National Natural Science Foundation of China (20175034, 20475059), and by the Chinese Academy of Sciences (KGCX2-SW-213-04).

\section{References}

1. Karas, M.; Hillenkamp, F. Laser Desorption Ionization of Proteins with Molecular Masses Exceeding 10,000 Daltons. Anal. Chem. 1988, 60, 2299-2301.

2. Karas, M.; Bachmann, D.; Bahr, U.; Hillenkamp, F. MatrixAssisted Ultraviolet Laser Desorption of Nonvolatile Compounds. Int. J. Mass Spectrom. Ion Processes 1987, 78, 53-68.

3. Karas, M.; Bachmann, D.; Hillenkamp, F. Influence of the Wavelength in High-Irradiance Ultraviolet Laser Desorption Mass Spectrometry of Organic Molecules. Anal. Chem. 1985, 57, 2935-2539.

4. Huberty, M. C.; Vath, J. E.; Yu, W.; Martin, S. A. Site-Specific Carbohydrate Identification in Recombinant Proteins Using MALDI-TOF MS. Anal. Chem. 1993, 65, 2791-2800.

5. Harvey, D. Matrix-Assisted Laser Desorption/Ionization Mass Spectrometry of Carbohydrates. J. Mass Spectrom. Rev. 1999, 18, 349-451.

6. Santos, L. S.; Henato, R.; Höehr, N. F.; Pilli, R. A.; Eberlin, M. N. Fast Screening of Low Molecular Weight Compounds by Thin-Layer Chromatography and "On-Spot" MALDI-TOF Mass Spectrometry. Anal. Chem. 2004, 76, 2144-2147.

7. Hoang, T. T.; Chen, Y. F.; May, S. W.; Browner, R. F. Analysis of Organoselenium Compounds in Human Urine Using Active Carbon and Chemically Modified Silica Sol-Gel SurfaceAssisted Laser Desorption/Ionization High-Resolution Timeof-Flight Mass Spectrometry. Anal. Chem. 2004, 76, 2062-2070.

8. Go, E. P.; Prenni, J. E.; Wei, J.; Jones, A.; Hall, S. C.; Witkowska, H. E.; Shen, Z;; Siuzdak, G. Desorption/Ionization on Silicon Time-of-Flight/Time-of-Flight Mass Spectrometry. Anal. Chem. 2003, 75, 2504-2506.

9. Cai, Y.; Jiang, Y. J.; Cole, R. B. Anionic Adducts of Oligosaccharides by Matrix-Assisted Laser Desorption/Ionization Time-of-Flight Mass Spectrometry. Anal. Chem. 2003, 75, 1638 1644. 
10. Cohen, L. H.; Gusev, A. I. Small Molecule Analysis by MALDI Mass Spectrometry. Anal. Bioanal. Chem. 2002, 373, 571-586.

11. Kim, H.-J.; Lee, J.-K.; Park, S.-J.; Ro, H. W.; Yoo, D. Y.; Yoon, D. Y. Observation of Low Molecular Weight Poly(methylsilsesquioxane)s by Graphite Plate Laser Desorption/Ionization Time-of-Flight Mass Spectrometry. Anal. Chem. 2000, 72, 56735678.

12. Ayorinde, F. O.; Garvin, K.; Saed, K. Determination of the Fatty Acid Composition of Saponified Vegetable Oils Using Matrix-Assisted Laser Desorption/Ionization Time-of-Flight Mass Spectrometry. Rapid Commun. Mass Spectrom. 2000, 14, 608-615.

13. Ayorinde, F. O.; Hambright, P.; Porter, T. N.; Keith, Q. L., Jr. Use of Meso-Tetrakis (Pentafluorophenyl)Porphyrin as a Matrix for Low Molecular Weight Alkylphenol Ethoxylates in Laser Desorption/Ionization Time-of-Flight Mass Spectrometry. Rapid Commun. Mass Spectrom. 1999, 13, 2474-2479.

14. Sunner, J.; Dratz, E.; Chen, Y.-C. Graphite Surface-Assisted Laser Desorption/Ionization Time-of-Flight Mass Spectrometry of Peptides and Proteins from Liquid Solutions. Anal. Chem. 1995, 67, 4335-4342.

15. Dale, M. J.; Konchenmuss, R.; Zenobi, R. Graphite/Liquid Mixed Matrices for Laser Desorption/Ionization Mass Spectrometry. Anal. Chem. 1996, 68, 3321-3329.

16. Chen, Y.-C.; Shiea, J.; Sunner, J. Rapid Determination of Trace Nitrophenolic Organic in Water by Combining Solid-Phase Extraction with Surface-Assisted Laser Desorption/Ionization Time-of Flight Mass Spectrometry. Rapid Commun. Mass Spectrom. 2000, 14, 86-90.

17. Kinumi, T.; Saisu, T.; Takayama, M.; Niwa, H. Matrix-Assisted Laser Desorption/Ionization Time-of-Flight Mass Spectrometry Using an Inorganic Particle Matrix for Small Molecule Analysis. J. Mass Spectrom. 2000, 35, 417-422.

18. Han, M.; Sunner, J. An Activated Carbon Substrate Surface for Laser Desorption Mass Spectrometry. J. AM. Soc. Mass. Spectrom 2000, 11, 644-649.

19. Michalak, L.; Fisher, K. J.; Alderdice, D. S.; Jardine, D. R.; Willett, G. D. $\mathrm{C}_{60}$-Assisted Laser Desorption-Ionization Mass Spectrometry. Org. Mass. Spectrom. 1994, 29, 512-515.

20. Wei, J.; Buriak, J. M.; Siuzdak, G. Desorption-Ionization Mass Spectrometry on Porous Silicon. Nature 1999, 399, 243-246.

21. Xu, S. Y.; Li, Y. F.; Zou, H. F.; Qiu, J. S.; Guo, Z.; Guo, B. C. Carbon Nanotubes as Assisted Matrix for Laser Desorption/
Ionization Time-of-Flight Mass Spectrometry. Anal. Chem. 2003, 75, 6191-6195.

22. Nicola A. J.; Gusev, A. I.; Proctor A.; Jackson E. K; Hercules D. M. Application of the Fast-Evaporation Sample Preparation Method for Improving Quantification of Angiotensin-II by Matrix-Assisted Laser-Desorption Ionization. Rapid Commun. Mass Spectrom. 1995, 9, 1164-1171.

23. Ekroos, K.; Chernushevich, I. V; Simons, K.; Shevchenko, A. Quantitative Profiling of Phospholipids by Multiple Precursor Ion Scanning on a Hybrid Quadrupole Time-of-Flight Mass Spectrometer. Anal. Chem. 2002, 74, 941-949.

24. Naven, T. J. P.; Harvey, D. J. Cationic Derivatization of Oligosaccharides with Girard's T Reagent for Improved Performance in Matrix-Assisted Laser Desorption/Ionization and Electrospray Mass Spectrometry. Rapid. Commun. Mass Spectrom. 1996, 10, 829-834.

25. Tondeur, Y. Role of the Matrix in the FAB MS Analysis of Monosaccharides. Org. Mass Spectrom. 1985, 20, 157-160.

26. Peter-Katalinić, J. Analysis of Glycoconjugates by Fast Atom Bombardment Mass Spectrometry and Related MS Techniques. Mass Spectrom. Rev. 1994, 13, 77-98.

27. Dallinga, J. W.; Heerma, W. Reaction Mechanism and Fragment Ion Structure Determination of Deprotonated Small Oligosaccharides, Studied by Negative Ion Fast Atom Bombardment (Tandem) Mass Spectrometry. Biol. Mass Spectrom. 1991, 20, 215-231.

28. Burlingame, A. L.; Baillie, T. A.; Derrick, P. J. Mass Spectrometry. Anal. Chem. 1986, 58, 165R-211R.

29. Barber, M.; Bordoli, R. S.; Sedgwick, R. D.; Tyler, A. N. Fast Atom Borbardment of Solids as an Ion Source in Mass Spectrometry. Nature 1981, 293, 270-275.

30. Gouw, J. W.; Burgers, P. C.; Trikoupis, M. A.; Terlouw, J. K. Derivatization of Small Oligosaccharides Prior to Analysis by Matrix-Assisted Laser Desorption/Ionization Using Glycidyltrimethylammonium Chloride and Girard's Reagent. Rapid Commun. Mass Spectrom. 2002, 16, 905-912.

31. Grant, G. A.; Frison, S. L. Vasanthan, T.; Sporns, P. Comparison of MALDI-TOF Mass Spectrometric to Enzyne Colorimetric Quantification of Glucose from Enzyme-Hydrolyzed Starch. J. Agric. Food Chem. 2003, 51, 6137-6144.

32. Mohr, M. D.; Börnsen, K. O.; Widmer, H. M. Matrix-Assisted Laser Desorption/Ionization Mass Spectrometry: Improved Matrix for Oligosaccharides. Rapid Commun. Mass Spectrom. $1995,9,809-814$ 